\title{
Some Properties of the Topological Entropy of a Family of Dynamical Systems Defined on an Arbitrary Metric Space
}

\author{
A. N. Vetokhin ${ }^{1,2 *}$ \\ ${ }^{1}$ Lomonosov Moscow State University, Moscow, 119991 Russia \\ ${ }^{2}$ Bauman Moscow State Technical University, Moscow, 105005 Russia \\ e-mail:*anveto27@yandex.ru \\ Received October 13, 2020; revised May 31, 2021; accepted June 8, 2021
}

\begin{abstract}
We consider a family of dynamical systems defined on a noncompact metric space and continuously depending on a parameter varying in some metric space. For any such family, the topological entropy of the dynamical systems in the family is studied as a function of the parameter from the viewpoint of the Baire classification of functions.
\end{abstract}

DOI: $10.1134 /$ S0012266121080012

The topological entropy of autonomous dynamical systems on an invariant compact metric space was defined in [1]. Later, this concept was extended in [2] to dynamical systems defined on an arbitrary metric space.

\section{BAIRE CLASS OF THE TOPOLOGICAL ENTROPY OF A FAMILY OF DYNAMICAL SYSTEMS IN THE CASE OF A NONINVARIANT COMPACT SET}

Following [2], let us give a definition needed in what follows. Let $(X, d)$ be a metric space, let $\mathcal{K}(X)$ be the set of compact subsets of $X$, and let $f: X \rightarrow X$ be a continuous mapping. Along with the original metric $d$, we define an additional system of metrics $d_{n}^{f}, n \in \mathbb{N}$, on $X$ by the formula

$$
d_{n}^{f}(x, y)=\max _{0 \leq i \leq n-1} d\left(f^{\circ i}(x), f^{\circ i}(y)\right), \quad x, y \in X, \quad n \in \mathbb{N},
$$

where $f^{\circ i}, i \in \mathbb{N}$, is the $i$ th iteration of $f$ and $f^{\circ 0} \equiv \operatorname{id}_{X}$. Given a $K \in \mathcal{K}(X)$, for any $n \in \mathbb{N}$ and $\varepsilon>0$ we denote by $N_{d}(K, f, \varepsilon, n)$ the maximum number of points in $K$ with pairwise $d_{n}^{f}$-distances greater than $\varepsilon$. The numbers

$$
\bar{h}_{\text {top }}(K, f)=\lim _{\varepsilon \rightarrow 0} \varlimsup_{n \rightarrow \infty} \frac{1}{n} \ln N_{d}(K, f, \varepsilon, n) \quad \text { and } \quad \underline{h}_{\text {top }}(K, f)=\lim _{\varepsilon \rightarrow 0} \frac{\lim }{n \rightarrow \infty} \frac{1}{n} \ln N_{d}(K, f, \varepsilon, n)
$$

are called the upper and lower topological entropies, respectively, of $f$ on $K$.

Note that if the metric $d$ is replaced by a metric generating the same topology as $d$, then the numbers (1) do not change [3, p. 121 of the Russian translation].

Let us also recall formulas for the upper and lower topological entropies, to be used below. For any $x \in X, \varepsilon>0$, and $n \in \mathbb{N}$, let $B_{f}(x, \varepsilon, n)$ be the open ball $\left\{y \in K: d_{n}^{f}(x, y)<\varepsilon\right\}$ of radius $\varepsilon$ centered at $x$ in the space $\left(X, d_{n}^{f}\right)$. A set $D \subset K$ is called an $(f, \varepsilon, n)$-cover of $K$ if

$$
K \subset \bigcup_{x \in D} B_{f}(x, \varepsilon, n) .
$$

Let $S_{d}(K, f, \varepsilon, n)$ be the minimum possible number of elements in an $(f, \varepsilon, n)$-cover of $K$. Then the upper and lower topological entropies of $f$ on $K$ can be calculated by the formulas [3, p. 122 of the Russian translation]

$$
\bar{h}_{\text {top }}(K, f)=\lim _{\varepsilon \rightarrow 0} \varlimsup_{n \rightarrow \infty} \frac{1}{n} \ln S_{d}(K, f, \varepsilon, n), \quad \underline{h}_{\mathrm{top}}(K, f)=\lim _{\varepsilon \rightarrow 0} \varliminf_{n \rightarrow \infty} \frac{1}{n} \ln S_{d}(K, f, \varepsilon, n) .
$$


Formulas (1) (or (2)) obviously imply the inequality $\bar{h}_{\text {top }}(K, f) \geq \underline{h}_{\text {top }}(K, f)$. If $K$ is $f$-invariant, i.e., $f(K) \subset K$, then the upper and lower topological entropies of $f$ on $K$ coincide [3, p. 122 of the Russian translation]. The following example shows that the numbers (1) do not necessarily coincide in the general case. Consider the set $\Omega_{2}$ of sequences $x=\left(x_{1}, x_{2}, x_{3}, \ldots\right)$, where $x_{i} \in\{0,1\}$, with the metric

$$
d_{\Omega_{2}}(x, y)= \begin{cases}0 & \text { if } x=y \\ 1 / \min \left\{i: x_{i} \neq y_{i}\right\} & \text { if } x \neq y\end{cases}
$$

Note that the space $\left(\Omega_{2}, d_{\Omega_{2}}\right)$ is homeomorphic to the Cantor set on the interval $[0,1]$ with the metric induced by the standard metric of the real line. Let $K_{0} \subset \Omega_{2}$ be the compact set defined by the condition

$$
\left(x_{1}, x_{2}, x_{3}, \ldots\right) \in K_{0} \Leftrightarrow x_{i}=0, \quad i \in \bigcup_{k \in \mathbb{N} \cup\{0\}}\{(2 k) !, \ldots,(2 k+1) !\}
$$

and let $\sigma: \Omega_{2} \rightarrow \Omega_{2}$ be the left shift mapping, $\sigma\left(\left(x_{1}, x_{2}, x_{3}, \ldots\right)\right)=\left(x_{2}, x_{3}, x_{4}, \ldots\right)$. Then

$$
\bar{h}_{\text {top }}\left(K_{0}, \sigma\right)=\ln 2, \quad \underline{h}_{\text {top }}\left(K_{0}, \sigma\right)=0 \text {. }
$$

Given a metric space $\mathcal{M}$, a compact set $K \subset X$, and a continuous mapping

$$
f: \mathcal{M} \times X \rightarrow X
$$

consider the functions

$$
\begin{aligned}
& \mu \mapsto \bar{h}_{\text {top }}(K, f(\mu, \cdot)), \\
& \mu \mapsto \underline{h}_{\text {top }}(K, f(\mu, \cdot)) .
\end{aligned}
$$

In the present paper, for each mapping (3) the functions (4) and (5) are studied from the viewpoint of the Baire classification of functions. Recall that continuous functions $M \rightarrow \mathbb{R}$ on a metric space $\mathcal{M}$ are called functions of Baire class 0 , and the functions of Baire class $p$ are defined for each positive integer $p$ as the functions that are pointwise limits of sequences of functions of Baire class $p-1$.

We have already mentioned that the numbers (1) coincide if $K$ is $f$-invariant; in that case, their common value is called the topological entropy of $f$ and is denoted by $h_{\text {top }}(f)$. In was established in the paper [4] that for each mapping (3) the function

$$
\mu \mapsto h_{\mathrm{top}}(f(\mu, \cdot))
$$

is of Baire class 2 on $\mathcal{M}$. In [5], a family of homeomorphisms (3) with $X=\mathcal{M}=\Omega_{2}$ was constructed such that the function (6) is not of Baire class 1; consequently, the functions (4) and (5), generally speaking, are not of Baire class 1 either. It was shown in [4] that if the space $\mathcal{M}$ is metrizable by a complete metric, then the set of points of lower semicontinuity of the function (6) contains a $G_{\delta}$ set everywhere dense in $\mathcal{M}$, while the paper [6] established that the set of points of lower semicontinuity itself is an everywhere dense $G_{\delta}$ set in $\mathcal{M}$. Further, let $X=\Omega_{2}$, and let $\mathcal{M}$ be an arbitrary complete metric separable zero-dimensional space (for example, $\Omega_{2}$ ). In this case, for an arbitrary $G_{\delta}$ set $\mathcal{G}$ everywhere dense in $\mathcal{M}$, the paper [7] presents the construction of a mapping (3) such that the set of points of lower semicontinuity of the corresponding function (6) coincides with $\mathcal{G}$. It turns our that the following assertion holds in the case of a noninvariant compact set $K$.

Theorem 1. For any $K \in \mathcal{K}(X)$ and any mapping (3), the function (4) is of Baire class 3 and the function (5) is of Baire class 2 on $\mathcal{M}$. If $\mathcal{M}$ is metrizable by a complete metric, then the set of points of lower semicontinuity of the function (5) is an everywhere dense $G_{\delta}$ set.

Proof. For any $\varepsilon>0$ and $n \in \mathbb{N}$, the function $\mu \mapsto n^{-1} \ln N_{d}(K, f(\mu, \cdot), \varepsilon, n)$ is lower semicontinuous [8] and the function $\mu \mapsto n^{-1} \ln S_{d}(K, f(\mu, \cdot), \varepsilon, n)$ is upper semicontinuous [4]; 
consequently [9, Ch. IX, Sec. 37, XI of the Russian translation], there exist sequences of continuous functions $\mu \mapsto \varphi_{d}^{m}(K ; \mu, \varepsilon, n)$ and $\mu \mapsto \psi_{d}^{m}(K ; \mu, \varepsilon, n), m \in \mathbb{N}$, on $\mathcal{M}$ such that

$$
\begin{aligned}
& \frac{1}{n} \ln N_{d}(K, f(\mu, \cdot), \varepsilon, n)=\sup _{m \in \mathbb{N}} \varphi_{d}^{m}(K ; \mu, \varepsilon, n), \quad \mu \in \mathcal{M}, \\
& \frac{1}{n} \ln S_{d}(K, f(\mu, \cdot), \varepsilon, n)=\inf _{m \in \mathbb{N}} \psi_{d}^{m}(K ; \mu, \varepsilon, n), \quad \mu \in \mathcal{M} .
\end{aligned}
$$

Hence we obtain the representations

$$
\begin{aligned}
\bar{h}_{\text {top }}(K, f(\mu, \cdot)) & =\lim _{\varepsilon \rightarrow 0} \varlimsup_{n \rightarrow \infty} \frac{1}{n} \ln N_{d}(K, f(\mu, \cdot), \varepsilon, n)=\sup _{k \in \mathbb{N}} \varlimsup_{n \rightarrow \infty} \sup _{m \in \mathbb{N}} \varphi_{d}^{m}(K ; \mu, 1 / k, n) \\
& =\sup _{k \in \mathbb{N}} \inf _{n \in \mathbb{N}} \sup _{l \geq n} \sup _{m \in \mathbb{N}} \varphi_{d}^{m}(K ; \mu, 1 / k, l) \\
& =\lim _{p \rightarrow \infty} \max _{1 \leq k \leq p} \lim _{r \rightarrow \infty} \min _{1 \leq n \leq r} \lim _{q \rightarrow \infty} \max _{n \leq l \leq q} \max _{1 \leq m \leq q} \varphi_{d}^{m}(K ; \mu, 1 / k, l)
\end{aligned}
$$

by (7) and

$$
\begin{aligned}
\underline{h}_{\text {top }}(K, f(\mu, \cdot)) & =\lim _{\varepsilon \rightarrow 0} \underline{\lim _{n \rightarrow \infty}} \frac{1}{n} \ln S_{d}(K, f(\mu, \cdot), \varepsilon, n)=\sup _{k \in \mathbb{N}} \underline{\lim }_{n \rightarrow \infty} \inf _{m \in \mathbb{N}} \psi_{d}^{m}(K ; \mu, 1 / k, n) \\
& =\sup _{k \in \mathbb{N}} \sup _{n \in \mathbb{N}} \inf _{l \geq n} \inf _{m \in \mathbb{N}} \psi_{d}^{m}(K ; \mu, 1 / k, l) \\
& =\lim _{p \rightarrow \infty} \max _{1 \leq k \leq p} \max _{1 \leq n \leq p} \lim _{q \rightarrow \infty} \min _{n \leq l \leq q} \min _{1 \leq m \leq q} \psi_{d}^{m}(K ; \mu, 1 / k, l)
\end{aligned}
$$

by (8).

Since the maximum and minimum of finitely many functions in a Baire class belong to the same class [9, Ch. IX, Sec. 37, III of the Russian translation], it obviously follows from these representations that the function $\mu \mapsto \bar{h}_{\text {top }}(K, f(\mu, \cdot))$ is of Baire class 3 and the function $\mu \mapsto \underline{h}_{\text {top }}(K, f(\mu, \cdot))$ is of Baire class 2 on $\mathcal{M}$.

Since the function $\mu \mapsto \underline{h}_{\text {top }}(K, f(\mu, \cdot))$ can be represented as the limit of a nondecreasing sequence of functions of Baire class 1 , we see that its set of points of lower semicontinuity is an everywhere dense $G_{\delta}$ set [10, Lemma 2]. The proof of the theorem is complete.

Note that if $\mathcal{M}$ is a complete metric space, then, by Baire's theorem [9, Ch. IX, Sec. 39, VI of the Russian translation], Theorem 1 implies that for each mapping (3) there exists an everywhere dense $G_{\delta}$ set $G \subset \mathcal{M}$ such that the restrictions of the functions $\mu \mapsto \underline{h}_{\text {top }}(K, f(\mu, \cdot))$ and $\mu \mapsto \bar{h}_{\text {top }}(K, f(\mu, \cdot))$ to $G$ are continuous.

There arises a natural question about the least Baire class containing the function (4). To answer this question, we construct metric spaces $\mathcal{B}$ and $\mathcal{C}$. By definition, the points of $\mathcal{B}$ are all possible (countable) sequences $\mu=\left(\mu_{k}\right)_{k=1}^{\infty}$ of positive integers. The distance between two points $\mu$ and $\nu$ is defined by the formula

$$
d_{\mathcal{B}}(\mu, \nu)= \begin{cases}0 & \text { if } \mu=\nu \\ 1 / \min \left\{k: \mu_{k} \neq \nu_{k}\right\} & \text { if } \mu \neq \nu .\end{cases}
$$

Note that the space $\left(\mathcal{B}, d_{\mathcal{B}}\right)$ is homeomorphic to the set of irrational numbers on the interval $[0,1]$ with the metric induced by the natural metric of the real line. The points of $\mathcal{C}$ are all possible pairs $(x, i)$, where $x \in[0,1]$ and $i \in \mathbb{N}$. The distance between points $(x, i)$ and $(y, j)$ is defined by the formula

$$
d_{\mathcal{C}}((x, i),(y, j))= \begin{cases}|x-y| & \text { if } i=j \\ 1 & \text { if } i \neq j .\end{cases}
$$

For each $r \in \mathbb{N}$, let $K_{r} \in \mathcal{K}(\mathcal{C})$ be the compact set $K_{r}=[0,1] \times\{1, \ldots, r\}$ in $\mathcal{C}$. 
Theorem 2. Let $\mathcal{M}=\mathcal{B}, X=\mathcal{C}$, and $K=K_{r}$; then there exists a mapping (3) such that the function (4) is everywhere discontinuous and does not belong to the second Baire class on $\mathcal{M}$.

Proof. Given a sequence $\mu=\left(\mu_{k}\right)_{k=1}^{\infty} \in \mathcal{B}$, we construct the sequence $\alpha(\mu)$ with elements $\alpha_{k}(\mu)=\mu_{\left[\log _{2}(k+1)\right]}$ (where [.] is the integer part of a number). Consider the sequence $\left(f_{k}\right)$ of mappings of $\mathcal{B} \times[0,1]$ into $[0,1]$ given by

$$
f_{k}(\mu, x)= \begin{cases}x & \text { if } 0 \leq x \leq 1-1 / \alpha_{k}(\mu) \\ 2 x-1+1 / \alpha_{k}(\mu) & \text { if } 1-1 / \alpha_{k}(\mu)<x \leq 1-1 /\left(2 \alpha_{k}(\mu)\right) \\ -2 x+3-1 / \alpha_{k}(\mu) & \text { if } 1-1 /\left(2 \alpha_{k}(\mu)\right)<x \leq 1\end{cases}
$$

We use this sequence to construct a mapping $f: \mathcal{B} \times \mathcal{C} \rightarrow \mathcal{C}$ by setting

$$
f(\mu,(x, k))=\left(f_{k}(\mu, x), k+1\right) .
$$

By definition, the function $f$ is continuous on $\mathcal{B} \times \mathcal{C}$.

Le $\mathcal{E} \subset \mathcal{B}$ be the set of sequences tending to infinity. Let us find the upper topological entropy of the mapping (9) for $\mu \in \mathcal{E}$.

Lemma 1. If $\mu \in \mathcal{E}$, then

$$
\bar{h}_{\text {top }}\left(K_{r}, f(\mu, \cdot)\right)=0
$$

for the mapping (9) for any $r \in \mathbb{N}$.

Proof. Fix an $\varepsilon \in(0,1)$ and a $\mu \in \mathcal{E}$. Then there exists a number $k_{0}(\varepsilon)>r$ such that $1 / \alpha_{k}(\mu)<\varepsilon / 2$ for each $k \geq k_{0}(\varepsilon)$.

Let $A_{k_{0}(\varepsilon)}$ be an $\left(f(\mu, \cdot), \varepsilon / 2, k_{0}(\varepsilon)\right)$-cover of $K_{r}$ with minimum number of elements. Let us prove that $A_{k_{0}(\varepsilon)}$ is an $\left(f(\mu, \cdot), \varepsilon, k_{0}(\varepsilon)+i\right)$-cover of $K_{r}$ for each $i \in \mathbb{N} \cup\{0\}$.

By the definition of $A_{k_{0}(\varepsilon)}$, for each point $(x, l) \in K_{r}$ there exists an element $\left(x_{0}, l\right) \in A_{k_{0}(\varepsilon)}$ such that $(x, l) \in B_{f(\mu, \cdot)}\left(\left(x_{0}, l\right), \varepsilon / 2, k_{0}(\varepsilon)\right)$.

If $f^{\circ\left(k_{0}(\varepsilon)-1\right)}(\mu,(x, l)), f^{\circ\left(k_{0}(\varepsilon)-1\right)}\left(\mu,\left(x_{0}, l\right)\right) \in[0,1-\varepsilon / 2] \times \mathbb{N}$, then

$$
\begin{aligned}
& d_{\mathcal{C}}\left(f^{\circ\left(k_{0}(\varepsilon)+i\right)}(\mu,(x, l)), f^{\circ\left(k_{0}(\varepsilon)+i\right)}\left(\mu,\left(x_{0}, l\right)\right)\right) \\
& \quad=d_{\mathcal{C}}\left(f^{\circ\left(k_{0}(\varepsilon)-1\right)}(\mu,(x, l)), f^{\circ\left(k_{0}(\varepsilon)-1\right)}\left(\mu,\left(x_{0}, l\right)\right)\right)<\varepsilon / 2
\end{aligned}
$$

for each $i \in \mathbb{N} \cup\{0\}$, because the interval $[0,1-\varepsilon / 2]$ is invariant with respect to the mapping $f_{k_{0}(\varepsilon)+i}(\mu, \cdot)$ for all $i \in \mathbb{N} \cup\{0\}$.

If $f^{\circ\left(k_{0}(\varepsilon)-1\right)}(\mu,(x, l)), f^{\circ\left(k_{0}(\varepsilon)-1\right)}\left(\mu,\left(x_{0}, l\right)\right) \in[1-\varepsilon / 2,1] \times \mathbb{N}$, then

$$
d_{\mathcal{C}}\left(f^{\circ\left(k_{0}(\varepsilon)+i\right)}(\mu,(x, l)), f^{\circ\left(k_{0}(\varepsilon)+i\right)}\left(\mu,\left(x_{0}, l\right)\right)\right) \leq \varepsilon / 2
$$

for each $i \in \mathbb{N} \cup\{0\}$, because the interval $[1-\varepsilon / 2,1]$ is invariant with respect to the mappings $f_{k_{0}(\varepsilon)+i}(\mu, \cdot)$ for all $i \in \mathbb{N} \cup\{0\}$.

If either $f^{\circ\left(k_{0}(\varepsilon)-1\right)}(\mu,(x, l)) \in[0,1-\varepsilon / 2] \times \mathbb{N}$ and $f^{\circ\left(k_{0}(\varepsilon)-1\right)}\left(\mu,\left(x_{0}, l\right)\right) \in[1-\varepsilon / 2,1] \times \mathbb{N}$ or $f^{\circ\left(k_{0}(\varepsilon)-1\right)}\left(\mu,\left(x_{0}, l\right)\right) \in[0,1-\varepsilon / 2] \times \mathbb{N}$ and $f^{\circ\left(k_{0}(\varepsilon)-1\right)}(\mu,(x, l)) \in[1-\varepsilon / 2,1] \times \mathbb{N}$, then

$$
\begin{aligned}
& d_{\mathcal{C}}\left(f^{\circ\left(k_{0}(\varepsilon)+i\right)}(\mu,(x, l)), f^{\circ\left(k_{0}(\varepsilon)+i\right)}\left(\mu,\left(x_{0}, l\right)\right)\right) \\
& \quad \leq d_{\mathcal{C}}\left(f^{\circ\left(k_{0}(\varepsilon)-1\right)}(\mu,(x, l)), f^{\circ\left(k_{0}(\varepsilon)-1\right)}\left(\mu,\left(x_{0}, l\right)\right)\right)+\varepsilon / 2<\varepsilon
\end{aligned}
$$

for each $i \in \mathbb{N} \cup\{0\}$. 
It follows from inequalities (10)-(12) that $(x, l) \in B_{f(\mu, \cdot)}\left(\left(x_{0}, l\right), \varepsilon, k_{0}(\varepsilon)+i\right)$ for each $i \in \mathbb{N} \cup\{0\}$, and hence the set $A_{k_{0}(\varepsilon)}$ is an $\left(f(\mu, \cdot), \varepsilon, k_{0}(\varepsilon)+i\right)$-cover of $K_{r}$ for each $i \in \mathbb{N} \cup\{0\}$. Thus, the estimate

$$
S_{d_{\mathcal{C}}}\left(K_{r}, f(\mu, \cdot), \varepsilon, n\right) \leq S_{d_{\mathcal{C}}}\left(K_{r}, f(\mu, \cdot), \varepsilon / 2, k_{0}(\varepsilon)\right)
$$

holds for $n \geq k_{0}(\varepsilon)$, which implies that

$$
\begin{aligned}
\bar{h}_{\mathrm{top}}\left(K_{r}, f(\mu, \cdot)\right) & =\lim _{\varepsilon \rightarrow 0} \varlimsup_{n \rightarrow \infty} \frac{1}{n} \ln S_{d_{\mathcal{C}}}\left(K_{r}, f(\mu, \cdot), \varepsilon, n\right) \\
& \leq \lim _{\varepsilon \rightarrow 0} \varlimsup_{n \rightarrow \infty} \frac{1}{n} \ln S_{d_{\mathcal{C}}}\left(K_{r}, f(\mu, \cdot), \varepsilon / 2, k_{0}(\varepsilon)\right)=0 .
\end{aligned}
$$

The proof of the lemma is complete.

Now let us estimate the upper topological entropy of the mapping (9) for $\mu \notin \mathcal{E}$.

Lemma 2. If $\mu \notin \mathcal{E}$, then

$$
\bar{h}_{\text {top }}\left(K_{1}, f(\mu, \cdot)\right) \geq \frac{1}{4} \ln 2
$$

for the mapping (9).

Proof. Let $\mu \notin \mathcal{E}$. Then there exists a subsequence $\left(\mu_{k_{j}}\right)_{j=1}^{\infty} \subset\left(\mu_{k}\right)_{k=1}^{\infty}$ and a positive integer $q$ such that $\mu_{k_{j}}=q$ for all $j \in \mathbb{N}$.

Further, $f_{k}(\mu, x)=f_{2^{k_{j}}-1}(\mu, x)=t_{q}(x)$ for all $j \in \mathbb{N}, k \in\left\{2^{k_{j}}-1, \ldots, 2^{k_{j}+1}-2\right\}$, and $x \in[0,1]$, where

$$
t_{q}(x)= \begin{cases}x & \text { if } 0 \leq x \leq 1-1 / q \\ 2 x-1+1 / q & \text { if } 1-1 / q<x \leq 1-1 /(2 q) \\ -2 x+3-1 / q & \text { if } 1-1 /(2 q)<x \leq 1\end{cases}
$$

The affine order-preserving transformation $\varphi$ mapping the interval $I_{q}=[1-1 / q, 1]$ onto the interval $[0,1]$ takes the mapping $\left.t_{q}\right|_{I_{q}}: I_{q} \rightarrow I_{q}$ to the mapping $g=\left.\varphi \circ t_{q}\right|_{I_{q}} \circ \varphi^{-1}:[0,1] \rightarrow[0,1]$ given by the formula

$$
g(x)= \begin{cases}2 x & \text { if } 0 \leq x \leq 1 / 2 \\ 2-2 x & \text { if } 1 / 2<x \leq 1\end{cases}
$$

It was established in the monograph [3, p. 502 of the Russian translation] that the topological entropy of $g$ is $\ln 2$; consequently, there exists an $\varepsilon_{0}<1 / q$ such that

$$
\underline{\lim _{n \rightarrow \infty}} \frac{1}{n} \ln N_{d}([0,1], g, \varepsilon, n) \geq \frac{1}{2} \ln 2, \quad d(x, y)=|x-y|,
$$

for every $\varepsilon<\varepsilon_{0}$. For each $n \in \mathbb{N}$, consider a set $\left\{a_{1}, \ldots, a_{N_{d}([0,1], g, \varepsilon, n)}\right\}$ of points on the interval $[0,1]$ with pairwise $d_{n}^{g}$-distances greater than $\varepsilon>0$.

Let $\varepsilon<\varepsilon_{0}$ and $n=2^{k_{j}+1}-2^{k_{j}}-1$. Then the $d_{2^{k_{j}+n-1}}^{f(\mu,)}$-distance between any preimages of two arbitrary points $\left(\varphi^{-1}\left(a_{i}\right), 2^{k_{j}}-1\right)$ and $\left(\varphi^{-1}\left(a_{m}\right), 2^{k_{j}}-1\right), i \neq m$, under the mapping $f^{\circ\left(2^{k_{j}}-2\right)}(\mu, \cdot)$ is greater than $\varepsilon / q>0$, and consequently,

$$
N_{d_{\mathcal{C}}}\left(K_{1}, f(\mu, \cdot), \varepsilon / q, 2^{k_{j}+1}\right) \geq N_{d}\left([0,1], g, \varepsilon, 2^{k_{j}+1}-2^{k_{j}}\right) .
$$

Hence we obtain the estimates

$$
\bar{h}_{\mathrm{top}}\left(K_{1}, f(\mu, \cdot)\right) \geq \lim _{\varepsilon \rightarrow 0} \varlimsup_{j \rightarrow \infty} \frac{\left(2^{k_{j}+1}-2^{k_{j}}\right)}{2^{k_{j}+1}} \frac{1}{\left(2^{k_{j}+1}-2^{k_{j}}\right)} \ln N_{d}\left([0,1], g, \varepsilon, 2^{k_{j}+1}-2^{k_{j}}\right) \geq \frac{1}{4} \ln 2 .
$$

The proof of the lemma is complete.

DIFFERENTIAL EQUATIONS Vol. $57 \quad$ No. $8 \quad 2021$ 
To complete the proof of Theorem 2, we use the following assertion established in [11]: if a function $\mu \mapsto \bar{h}_{\text {top }}\left(K_{r}, f(\mu, \cdot)\right)$ is of Baire class 2, then the intersection of closures of the sets $\bar{h}_{\text {top }}\left(K_{r}, f(\mathcal{E}, \cdot)\right)$ and $\bar{h}_{\text {top }}\left(K_{r}, f(\mathcal{B} \backslash \mathcal{E}, \cdot)\right)$ is nonempty. By Lemmas 1 and 2 ,

$$
\bar{h}_{\text {top }}\left(K_{r}, f(\mathcal{E}, \cdot)\right)=0<\frac{1}{4} \ln 2 \leq \bar{h}_{\text {top }}\left(K_{1}, f(\mathcal{B} \backslash \mathcal{E}, \cdot)\right) \leq \bar{h}_{\text {top }}\left(K_{r}, f(\mathcal{B} \backslash \mathcal{E}, \cdot)\right),
$$

and consequently, the function $\mu \mapsto \bar{h}_{\text {top }}\left(K_{r}, f(\mu, \cdot)\right)$ is not of Baire class 2. Since both $\mathcal{E}$ and $\mathcal{B} \backslash \mathcal{E}$ are everywhere dense in $\mathcal{B}$, it follows that this function is everywhere discontinuous on $\mathcal{B}$. The proof of the theorem is complete.

\section{BAIRE CLASS OF THE TOPOLOGICAL ENTROPY OF A FAMILY OF DYNAMICAL SYSTEMS ON A NONCOMPACT METRIC SPACE}

Following [2], we define the upper and lower topological entropies of a mapping $f: X \rightarrow X$ as the numbers

$$
\bar{h}_{\mathrm{top}}(f)=\sup _{K \in \mathcal{K}(X)} \bar{h}_{\mathrm{top}}(K, f), \quad \underline{h}_{\mathrm{top}}(f)=\sup _{K \in \mathcal{K}(X)} \underline{h}_{\mathrm{top}}(K, f),
$$

respectively. The following example shows that the two numbers (13) may be distinct. Let us construct a space $\mathcal{A}$ as follows. The points of $\mathcal{A}$ are all possible pairs $(x, i)$, where $x \in \Omega_{2}$ and $i \in \mathbb{N}$, and the metric is defined by the formula

$$
d_{\mathcal{A}}((x, i),(y, j))= \begin{cases}d_{\Omega_{2}}(x, y) & \text { if } i=j \\ 1 & \text { if } i \neq j\end{cases}
$$

Consider the sequence

$$
f_{n}=\left\{\begin{array}{ll}
\operatorname{id}_{\Omega_{2}} & \text { if } t_{2 k} \leq n \leq t_{2 k+1}-1 \\
\sigma & \text { if } t_{2 k+1} \leq n \leq t_{2 k+2}-1,
\end{array} \quad t_{s}=\sum_{m=0}^{s} m !, \quad k=0,1,2, \ldots,\right.
$$

of continuous self-mappings of $\Omega_{2}$ and define a continuous mapping $f_{\mathcal{A}}: \mathcal{A} \rightarrow \mathcal{A}$ by setting

$$
f_{\mathcal{A}}(x, n)=\left(f_{n}(x), n+1\right) .
$$

Lemma 3. $\underline{h}_{\text {top }}\left(f_{\mathcal{A}}\right)<\bar{h}_{\text {top }}\left(f_{\mathcal{A}}\right)$.

Proof. For an arbitrary $r \in \mathbb{N}$, let $H_{r} \in \mathcal{K}(\mathcal{A})$ be the compact set $H_{r}=\Omega_{2} \times\{1, \ldots, r\}$. Each compact set $K \in \mathcal{K}(\mathcal{A})$ is contained in $H_{r}$ for some $r$; consequently,

$$
\underline{h}_{\text {top }}\left(f_{\mathcal{A}}\right)=\lim _{r \rightarrow \infty} \underline{h}_{\text {top }}\left(H_{r}, f_{\mathcal{A}}\right), \quad \bar{h}_{\text {top }}\left(f_{\mathcal{A}}\right)=\lim _{r \rightarrow \infty} \bar{h}_{\text {top }}\left(H_{r}, f_{\mathcal{A}}\right) \text {. }
$$

Let $p \in \mathbb{N}$, and let $Q$ be an $\left(f_{\mathcal{A}}, 1 / p, t_{2 k}\right)$-cover of $H_{r}$ with minimum number of elements. Then $Q$ is an $\left(f_{\mathcal{A}}, 1 / p, t_{2 k+1}\right)$-cover of $H_{r}$ by the definition of $f_{\mathcal{A}}$. Since the points $(x, i) \in \mathcal{A}$, where $x=\left(x_{1}, \ldots, x_{t_{2 k}+p}, 0,0, \ldots\right)$ and $i \in\{1,2, \ldots, r\}$, form an $\left(f_{\mathcal{A}}, 1 / p, t_{2 k}\right)$-cover of $H_{r}$, it follows that the number of elements in $Q$ does not exceed $r 2^{t_{2 k}+p}$. Therefore,

$$
\underline{h}_{\text {top }}\left(H_{r}, f_{\mathcal{A}}\right) \leq \lim _{p \rightarrow \infty} \lim _{k \rightarrow \infty}\left(\frac{t_{2 k}+p}{(2 k+1) !} \ln 2+\frac{\ln r}{(2 k+1) !}\right) \leq \lim _{k \rightarrow \infty} \frac{\ln 2}{2 k+1}\left(2+\frac{p}{(2 k) !}\right)=0,
$$

and hence $\underline{h}_{\text {top }}\left(f_{\mathcal{A}}\right)=0$.

Let us establish the inequality $\bar{h}_{\text {top }}\left(f_{\mathcal{A}}\right) \geq 0.5 \ln 2$, whence Lemma 3 will follow. In the space $\Omega_{2}$, consider the set $R_{k}, k \in \mathbb{N} \cup\{0\}$, of points of the form

$$
\left(x_{1}, \ldots, x_{(2 k+2) !}, 0,0, \ldots\right) .
$$


Take one point $\left(y_{x}, 1\right) \in H_{1}$ in the preimage of every point $\left(x, t_{2 k+1}\right), x \in R_{k}$, under the mapping $f_{\mathcal{A}}^{\circ\left(t_{2 k+1}-1\right)}$. If $x^{\prime} \neq x^{\prime \prime}, x^{\prime}, x^{\prime \prime} \in R_{k}$, then

$$
d_{t_{2 k+2}}^{f_{\mathcal{A}}}\left(\left(y_{x^{\prime}}, 1\right),\left(y_{x^{\prime \prime}}, 1\right)\right) \geq \max _{0 \leq i \leq(2 k+2) !-1} d_{\mathcal{A}}\left(f_{\mathcal{A}}^{\circ i}\left(x^{\prime}, t_{2 k+1}\right), f_{\mathcal{A}}^{\circ i}\left(x^{\prime \prime}, t_{2 k+1}\right)\right)=1 .
$$

Thus, $N_{d_{\mathcal{A}}}\left(H_{1}, f_{\mathcal{A}}, \varepsilon, t_{2 k+2}\right)$ is not less than the cardinality of $R_{k}$, which is $2^{(2 k+2) !}$, for each $\varepsilon<1$, and hence

$$
\bar{h}_{\text {top }}\left(f_{\mathcal{A}}\right) \geq \bar{h}_{\text {top }}\left(H_{1}, f_{\mathcal{A}}\right) \geq \lim _{\varepsilon \rightarrow 0} \varlimsup_{k \rightarrow \infty} \frac{1}{t_{2 k+2}} \ln N_{d_{\mathcal{A}}}\left(H_{1}, f_{\mathcal{A}}, \varepsilon, t_{2 k+2}\right) \geq \lim _{k \rightarrow \infty} \frac{(2 k+2) !}{t_{2 k+2}} \ln 2 \geq \frac{\ln 2}{2} .
$$

The proof of the lemma is complete.

For the mapping (3), consider the functions

$$
\begin{aligned}
& \mu \mapsto \bar{h}_{\text {top }}(f(\mu, \cdot)), \\
& \mu \mapsto \underline{h}_{\text {top }}(f(\mu, \cdot)) .
\end{aligned}
$$

If $X$ is a compact metric space, then the numbers (13) are equal to the topological entropy of $f$. Therefore, the functions (14) and (15) are of Baire class 2 by [4] but in general not of Baire class 1 by [5].

Theorem 3. If $\mathcal{M}=\mathcal{B}$ and $X=\mathcal{C}$, then there exists a mapping (3) such that the function (14) is everywhere discontinuous and does not belong to the second Baire class on $\mathcal{M}$.

Proof. Each compact set $K \in \mathcal{K}(\mathcal{C})$ is contained in $K_{r}$ for some $r$, and consequently, the topological entropy of an arbitrary continuous mapping $f: \mathcal{C} \rightarrow \mathcal{C}$ satisfies the relation

$$
\bar{h}_{\text {top }}(f)=\sup _{K \in \mathcal{K}(\mathcal{C})} \bar{h}_{\text {top }}(K, f)=\sup _{r \in \mathbb{N}} \bar{h}_{\text {top }}\left(K_{r}, f\right) .
$$

By Lemmas 1 and 2, we obtain the chain of inequalities

$$
\bar{h}_{\text {top }}(f(\mathcal{E}, \cdot))=0<\frac{1}{4} \ln 2 \leq \bar{h}_{\text {top }}(f(\mathcal{B} \backslash \mathcal{E}, \cdot))
$$

for the family (9). Consequently, the function $\mu \mapsto \bar{h}_{\text {top }}(f(\mu, \cdot))$ is not of Baire class 2 [11], and since the sets $\mathcal{E}$ and $\mathcal{B} \backslash \mathcal{E}$ are everywhere dense in $\mathcal{B}$, it follows that this function is everywhere discontinuous on $\mathcal{B}$. The proof of the theorem is complete.

Recall that a metric space $X$ is said to be locally compact if each of its points has a compact neighborhood [12, p. 315 of the Russian translation]. A locally compact space $X$ is said to be countable at infinity [12, p. 316 of the Russian translation] if it is a union of a countably many compact sets. The space $\mathbb{R}^{n}$, the above-defined space $\mathcal{C}$, and, in general, any locally compact space with countable base are examples of such spaces [12, p. 316; 13, p. 254].

Theorem 4. Let $X$ be a locally compact space countable at infinity. Then, for any space $\mathcal{M}$ and any mapping (3), the function (14) is of Baire class 3 on $\mathcal{M}$ and the function (15) is of Baire class 2 on $\mathcal{M}$. If $\mathcal{M}$ is metrizable by a complete metric, then, for each mapping (3), the set of points of lower semicontinuity of the function (15) is a $G_{\delta}$ set everywhere dense in $\mathcal{M}$.

Proof. Since $X$ is countable at infinity, it follows that there exists an increasing sequence $\left\{U_{s}\right\}_{s=1}^{\infty}$ of relatively compact open sets that form a cover of $X$ and satisfy $\bar{U}_{s} \subset U_{s+1}$ for all $s \in \mathbb{N}[12$, p. 316 of the Russian translation]. Every compact set $K \subset X$ is contained in $U_{s_{0}}$ for some $s_{0}$, because otherwise the cover of $K$ by the sequence $\left\{U_{s}\right\}_{s=1}^{\infty}$ would not contain a finite subcover, which contradicts the compactness of $K$. Thus,

$$
\begin{aligned}
& \bar{h}_{\mathrm{top}}(f)=\sup _{K \in \mathcal{K}(X)} \bar{h}_{\mathrm{top}}(K, f)=\sup _{s \in \mathbb{N}} \bar{h}_{\mathrm{top}}\left(\bar{U}_{s}, f\right), \\
& \underline{h}_{\mathrm{top}}(f)=\sup _{K \in \mathcal{K}(X)} \underline{h}_{\mathrm{top}}(K, f)=\sup _{s \in \mathbb{N}} \underline{h}_{\mathrm{top}}\left(\bar{U}_{s}, f\right)
\end{aligned}
$$

DIFFERENTIAL EQUATIONS Vol. $57 \quad$ No. $8 \quad 2021$ 
for each continuous mapping $f: X \rightarrow X$. Using formula (7), we obtain

$$
\begin{aligned}
\bar{h}_{\text {top }}(f(\mu, \cdot)) & =\sup _{s \in \mathbb{N}} \lim _{\varepsilon \rightarrow 0} \varlimsup_{n \rightarrow \infty} \frac{1}{n} \ln N_{d}\left(\bar{U}_{s}, f(\mu, \cdot), \varepsilon, n\right) \\
& =\sup _{s \in \mathbb{N}} \sup _{k \in \mathbb{N}} \varlimsup_{n \rightarrow \infty} \sup _{m \in \mathbb{N}} \varphi_{d}^{m}\left(\bar{U}_{s} ; \mu, 1 / k, n\right)=\sup _{s \in \mathbb{N}} \sup _{k \in \mathbb{N}} \inf _{n \in \mathbb{N}} \sup _{l \geq n} \sup _{m \in \mathbb{N}} \varphi_{d}^{m}\left(\bar{U}_{s} ; \mu, 1 / k, l\right) \\
& =\lim _{p \rightarrow \infty} \max _{1 \leq s \leq p} \max _{1 \leq k \leq p} \lim _{r \rightarrow \infty} \min _{1 \leq n \leq r} \lim _{q \rightarrow \infty} \max _{n \leq l \leq q} \max _{1 \leq m \leq q} \varphi_{d}^{m}\left(\bar{U}_{s} ; \mu, 1 / k, l\right) .
\end{aligned}
$$

By (8), we have

$$
\begin{aligned}
\underline{h}_{\mathrm{top}}(f(\mu, \cdot)) & =\sup _{s \in \mathbb{N}} \lim _{\varepsilon \rightarrow 0} \underline{\lim } \frac{1}{n \rightarrow \infty} \ln S_{d}\left(\bar{U}_{s}, f(\mu, \cdot), \varepsilon, n\right) \\
& =\sup _{s \in \mathbb{N}} \sup _{k \in \mathbb{N}} \lim _{n \rightarrow \infty} \inf _{m \in \mathbb{N}} \psi_{d}^{m}\left(\bar{U}_{s} ; \mu, 1 / k, n\right)=\sup _{s \in \mathbb{N}} \sup _{k \in \mathbb{N}} \sup _{n \in \mathbb{N}} \inf _{l \geq n} \inf _{m \in \mathbb{N}} \psi_{d}^{m}\left(\bar{U}_{s} ; \mu, 1 / k, l\right) \\
& =\lim _{p \rightarrow \infty} \max _{1 \leq s \leq p} \max _{1 \leq k \leq p} \max _{1 \leq n \leq p} \lim _{q \rightarrow \infty} \min _{n \leq l \leq q} \min _{1 \leq m \leq q} \psi_{d}^{m}\left(\bar{U}_{s} ; \mu, 1 / k, l\right) .
\end{aligned}
$$

Since the maximum and minimum of finitely many functions in some Baire class belong to the same class $\left[9\right.$, Ch. IX, Sec. 37, III of the Russian translation], it follows that the function $\mu \mapsto \bar{h}_{\text {top }}(f(\mu, \cdot))$ is of Baire class 3 and the function $\mu \mapsto \underline{h}_{\text {top }}(f(\mu, \cdot))$ is of Baire class 2 on $\mathcal{M}$.

Since the function $\mu \mapsto \underline{h}_{\text {top }}(f(\mu, \cdot)$,$) can be represented as the limit of a nondecreasing sequence$ of functions of Baire class 1, it follows that its set of points of lower semicontinuity is an everywhere dense $G_{\delta}$ set provided that $\mathcal{M}$ is metrizable by a complete metric [10, Lemma 2]. The proof of the theorem is complete.

\section{OPEN ACCESS}

This article is licensed under a Creative Commons Attribution 4.0 International License, which permits use, sharing, adaptation, distribution and reproduction in any medium or format, as long as you give appropriate credit to the original author(s) and the source, provide a link to the Creative Commons license, and indicate if changes were made. The images or other third party material in this article are included in the article's Creative Commons license, unless indicated otherwise in a credit line to the material. If material is not included in the article's Creative Commons license and your intended use is not permitted by statutory regulation or exceeds the permitted use, you will need to obtain permission directly from the copyright holder. To view a copy of this license, visit http://creativecommons.org/licenses/by/4.0/.

\section{REFERENCES}

1. Adler, R.L., Konheim, A.G., and McAndrew, M.H., Topological entropy, Trans. Am. Math. Soc., 1965, vol. 114, pp. 309-319.

2. Bowen, R., Entropy for group endomorphisms and homogeneous spaces, Trans. Am. Math. Soc., 1971, vol. 153, pp. 401-414.

3. Katok, A. and Hasselblatt, B., Introduction to the Modern Theory of Dynamical Systems, Cambridge: Cambridge Univ. Press, 1999. Translated under the title: Vvedenie v sovremennuyu teoriyu dinamicheskikh sistem, Moscow: Faktorial, 1999.

4. Vetokhin, A.N., Typical property of the topological entropy of continuous mappings of compact sets, Differ. Equations, 2017, vol. 53, no. 4, pp. 439-444.

5. Vetokhin, A.N., The topological entropy on a space of homeomorphisms does not belong to the first Baire class, Moscow Univ. Math. Bull., 2016, vol. 71, pp. 75-78.

6. Vetokhin, A.N., The set of lower semi-continuity points of topological entropy of a continuous oneparametric family of dynamical systems, Moscow Univ. Math. Bull., 2019, vol. 74, pp. 131-133.

7. Vetokhin, A.N., On some properties of topological entropy and topological pressure of families of dynamical systems continuously depending on a parameter, Differ. Equations, 2019, vol. 55, no. 10, pp. $1275-1283$.

8. Vetokhin, A.N., The exact Baire class of topological entropy of nonautonomous dynamical systems, Math. Notes, 2019, vol. 106, no. 3, pp. 327-333. 
9. Hausdorff, F., Mengenlehre, Berlin-Leipzig: de Gruyter, 1935. Translated under the title: Teoriya mnozhestv, Moscow-Leningrad: Gl. Red. Fiz.-Mat. Lit., 1937.

10. Karpuk, M.V., Construction of the set of the points of semicontinuity of Lyapunov exponents for linear differential systems continuously depending on a parameter, Differ. Uravn., 2015, vol. 51, no. 10, pp. 1404-1408.

11. Vetokhin, A.N., Baire class of the maximum lower semicontinuous minorants of Lyapunov exponents, Differ. Uravn., 1998, vol. 34, no. 10, pp. 1313-1317.

12. Bourbaki, N., Éléments de mathématique. Topologie générale, Paris: Hermann, 1939. Translated under the title: Obshchaya topologiya. Ispol'zovanie veshchestvennykh chisel v obshchei topologii. Funktsional'nye prostranstva. Svodka rezul'tatov. Slovar', Moscow: Gl. Red. Fiz.-Mat. Lit., 1975.

13. Nemytskii, V.V. and Stepanov, V.V., Kachestvennaya teoriya differentsial'nykh uravnenii (Qualitative Theory of Differential Equations), Moscow-Leningrad: Gos. Izd. Tekh.-Teor. Lit., 1949. 